\title{
BM] open Quality and safety in transitional care of the elderly: the study protocol of a case study research design (phase 1)
}

\author{
Karina Aase, ${ }^{1,2}$ Kristin Alstveit Laugaland, ${ }^{3}$ Dagrunn Nåden Dyrstad, ${ }^{1,4}$ \\ Marianne Storm ${ }^{1}$
}

To cite: Aase $\mathrm{K}$ Laugaland KA, Dyrstad DN, et al. Quality and safety in transitional care of the elderly: the study protocol of a case study research design (phase 1). BMJ Open 2013;3:e003506. doi:10.1136/bmjopen-2013003506

- Prepublication history for this paper is available online. To view these files please visit the journal online (http://dx.doi.org/10.1136/ bmjopen-2013-003506)

Received 29 June 2013 Accepted 4 July 2013

\footnotetext{
${ }^{1}$ Department of Health Studies, University of Stavanger, Stavanger, Norway ${ }^{2}$ Regional Centre for Agerelated Medicine, Stavanger University Hospital, Stavanger, Norway ${ }^{3}$ Førde Central Hospital, Førde, Norway

${ }^{4}$ Department of Anaesthesiology and Intensive Care, Stavanger University Hospital, Stavanger, Norway
}

Correspondence to Dr Karina Aase; karina.aase@uis.no

\begin{abstract}
Introduction: Although international studies have documented that patients' transitions between care providers are associated with the risk of adverse events and uncoordinated care, research directed towards the quality and safety of transitional care between primary and secondary health and care services, especially for the elderly receiving care from multiple healthcare providers due to complex health problems, is lacking. This study investigates how different aspects of transitional care can explain the quality and safety of elderly healthcare services in Norway. The overall aim of the study was to explore different aspects of transitional care of the elderly, in different contexts and how they might explain the quality and safety of care.

Methods and analysis: The study applies a case study design. Two cases are chosen: one city-based hospital and one rural hospital with associated nursing homes and home-based nursing services. Admission and discharge to/from hospital to/from nursing homes or home-based nursing services constitute the main focal areas of the study, including the patient, next-ofkin and the professional perspective. The qualitative methods employed include participant observation, individual interviews and document analysis. To ensure trustworthiness in the data analysis, we will apply analyst triangulation and member checks. A total impression of the data material will first be created in a systematic text condensation approach. Second, the qualitative data analysis will involve in-depth analyses of two specific themes: the risk perspective and the patient perspective in transitional care.
\end{abstract}

Ethics and dissemination: The study is approved by the Norwegian Regional Committees for Medical and Health Research Ethics. The study is based on informed written consent, and informants can withdraw from the study at any point in time. Interview and observation data material will be managed confidentially.

Results: It will be disseminated at research conferences, in peer-reviewed journals and through public presentations to people outside the academic community.

\section{INTRODUCTION}

Transitional care has become one of the most pressing topics in the global efforts to improve the quality and safety of patients due to the growing evidence indicating a correlation of patient handovers with medical errors and adverse events. Transitional care in this setting is defined as a set of actions ensuring the coordination and continuity of healthcare as patients transfer among different locations and different levels of care within the same location. ${ }^{1}$ The transfer of essential information and the responsibility for the care of the patient from one healthcare provider to another are integral and vital components of quality and safety in healthcare services.

The elderly population with complex health problems typically receives care from numerous healthcare providers and moves frequently within and across healthcare settings. A growing body of evidence suggests that the elderly are particularly vulnerable to experiencing discontinuity in care with the potential for adverse outcomes due to poorly executed transitions. ${ }^{1-4}$ Elderly patients, many with reduced mental capacity, are those most dependent on a healthcare system that is able to communicate appropriately and transfer information and duties properly. ${ }^{5}$ Frail elderly patients, particularly those with cognitive impairment, consistently suffer repeated hospitalisation, iatrogenic complications and uncoordinated care. ${ }^{6}$ A review of the growing body of literature reveals that relatively little data are available to document quality and safety-related issues occurring during the transitions that interface between primary and secondary healthcare services. ${ }^{7}$ Thus far, little data have been available to estimate the breaches to quality and safety in the posthospital period. ${ }^{8}$ A commendable exception is the recent HANDOVER study, a large multicentre and multinational study on patient transitions from the acute hospital to the primary care setting using a mixed-methods approach involving patients and care providers. ${ }^{9}$ 
The Coordination Reform, launched in 2009, is the principal healthcare reform implemented in Norway from 1 January 2012. The reform aims to solve three primary challenges in the Norwegian healthcare services: (1) patients' needs for co-ordinated services, (2) increased attention on disease prevention and (3) population development and the changing range of illnesses among the population. The Coordination Reform further accentuates the relevance of the 'Quality and Safety in Transitional Care of the Elderly' study as it implies changes to the contextual setting in which the transitional care of the elderly takes place.

\section{AIMS}

The 'Quality and Safety in Transitional Care of the Elderly' study has two main objectives:

1. To explore different aspects of transitional care of the elderly (eg, coordination, multiprofessional collaboration, patient participation) in different contexts (eg, admission or discharge, densely or sparsely populated geographical areas) and how they might explain the quality and safety of care (phase 1).

2. To design and test an evidence-based intervention programme to assess the impact of transitional care on quality and safety and to implement improvements within the transitional care of the elderly (phase 2).

This study protocol covers phase 1 of the project.

Thus far, most studies on transitional care (handovers, handoffs, etc) have employed methods such as individual or focus group interviews with patients and professionals ${ }^{10-14}$ and, to a much lesser extent, methods involving the real-time observation of practice when patients cross care provider boundaries. Real-time observational studies have been more common within, for instance, anaesthesia and surgery, ${ }^{15}$ where different methods and techniques have been employed in the study of behaviour of, for instance, operating theatre teams. Here, the focus is the professional perspective as the patient plays a passive role, having been anaesthetised. A clinician-centred approach has also been the focus of different observational studies of handovers at hospitals, often with the aim of mapping information dynamics and communication processes. ${ }^{16}{ }^{17}$ As part of the patient-centred care movement, techniques such as patient and family shadowing have been developed within a more practice-based improvement or change perspective. ${ }^{18}$ The aim of such efforts is to have an observer follow a patient and family throughout a selected care experience (often in-hospital surgery or trauma care) to view and capture the details of the entire care experience from the point of view of the patient and family.

In the 'Quality and Safety in Transitional Care of the Elderly' study, real-time observations of transitional care practice constitute the main data material (supported by structured interviews and document analyses), combining the professional perspective and the patient perspective by including patients, next of kin and care providers in observational case studies.

\section{METHODS AND ANALYSIS}

The 'Quality and Safety in Transitional Care of the Elderly' study (phase 1) employs a case study research design using multiple qualitative methods. Case studies are a preferred design within complex contexts where it is difficult to isolate variables or where strong interactions occur among variables, ${ }^{19}$ which is particularly relevant for this study of transitional care involving multiple contexts and variables. Two cases are chosen based on a most dissimilar strategy, where a case consists of one hospital along with its associated nursing homes and homebased nursing services:

- Case A consists of a small rural hospital (approximately 2000 employees) and three relatively small rural nursing homes with associated home care services in three municipalities.

- Case $B$ consists of a relatively large city-based university hospital (approximately 7000 employees) and three relatively large city-based nursing homes with associated home care services in one municipality.

Both cases are situated in the same Regional Health Authority in Norway.

The aim of the case study research design was fourfold: (1) to explore the phenomenon of transitional care; (2) to become familiar with each case as a stand-alone entity, allowing unique patterns of each case to emerge; (3) to conduct cross-case comparisons, searching for patterns across the cases (similarities and differences) and (4) to contribute to the development of context-specific theories of transitional care and the factors influencing quality and safety in transitional care.

Two types of transitions will be studied in case A and case $\mathbf{B}$, as illustrated in figure 1 . The transitions included are the admission of elderly patients to the hospital from the nursing home or home with home-based care services and the discharge of elderly patients from the hospital to the nursing home or home with home-based care services.

The admission transitions included are acute admissions of the elderly from nursing homes or home with home-based care services to the emergency department at the hospital via ambulance or the municipal emergency clinic. The discharge transitions included are the discharge of elderly patients from different hospital departments via ambulance or taxi transport to nursing homes or home with home-based care services.

The overarching research questions guiding the study of transitional care in the two case studies are as follows:

A. How are admission and discharge transitions of the elderly across primary (nursing homes, home-based services, general practitioners) and secondary (hospitals) care providers carried out?

B. How can different aspects of admission and discharge transitions of the elderly (eg, coordination, 


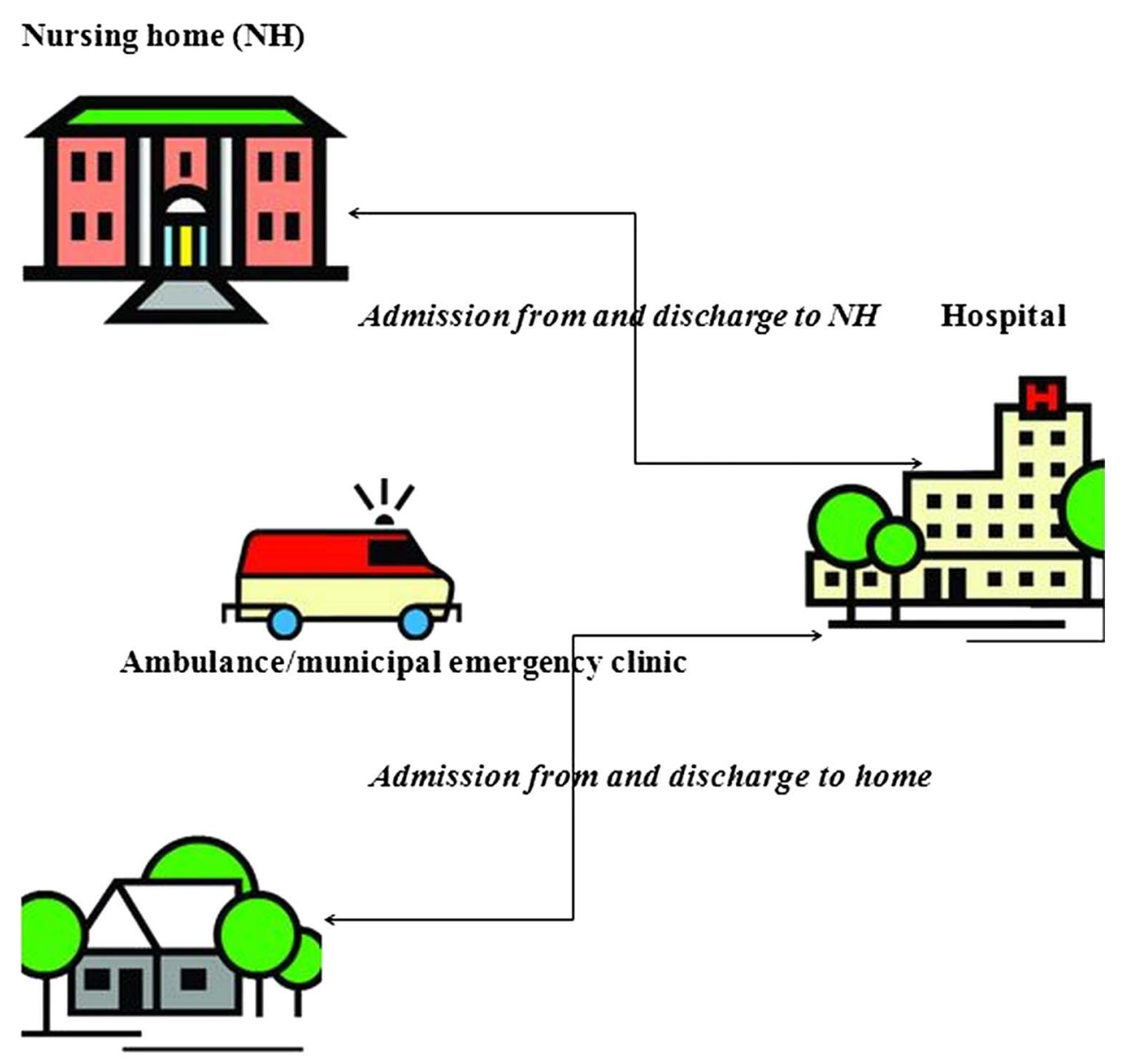

\section{Home with home-based care services}

Figure 1 Transitions included in the study.

multiprofessional collaboration, patient participation) explain the quality of transitional care across primary and secondary care providers?

C. What are the risks associated with admission and discharge transitions of the elderly across primary and secondary care providers?

D. How is the patient perspective (patients and next of kin) embedded in admission and discharge transitions of the elderly across primary and secondary care providers?

E. What measures need to be initiated to ensure quality and safety in admission and discharge transitions of the elderly across primary and secondary care providers?

\section{Study sample}

The main aim of phase 1 of the 'Quality and Safety in Transitional Care of the Elderly' project is to explore different aspects of transitional care of the elderly in different contexts. Including admission and discharge and employing the most dissimilar case strategy (case A and case B) addresses the issue of different aspects and contexts. Concerning the elderly group, we focus on the frail elderly ${ }^{\mathrm{i}}(>75$ years old). Our literature review demonstrated that few studies exist concerning the quality and safety of frail elderly patients. ${ }^{7}$

Within the frail elderly group, we want to include patients with different diagnoses covering orthopaedic and medical conditions. We also want to cover patients with poly-pharmacy ( $>5$ medications), given the knowledge that adverse events in transitional care of the elderly are often associated with medication errors. ${ }^{7}$ In Norway, approximately $50 \%$ of the elderly ( $>70$ years old) receive prescriptions for more than five medications, and $20 \%$ receive them for more than 10 medications. ${ }^{21}$ Within orthopaedic conditions, the frail elderly are at high risk of hip fracture (upper femur); in

${ }^{\mathrm{i}}$ One of several definitions of the frail elderly is " $A$ person older than 75 years of age who has been hospitalized three or more times in the last 12 months and has three or more diagnoses in their medical records according to the International Classification of Diseases (ICD-10)" ${ }^{20}$ In this study, we seek to include patients according to such a definition of frail elderly in parts of the study sample. 
Norway, 9000 elderly are admitted to hospital with this diagnosis each year. ${ }^{22}$

Hip fracture represents a marker for vulnerability and is often associated with trauma (bleeding, pain, loss of function, increased care need) for the elderly patient. ${ }^{23}$ Among the medical conditions, chronic obstructive pulmonary disease (COPD) is a diagnosis that increases among the elderly with a high mortality rate. ${ }^{24}$ In Norway, the elderly with COPD have a significantly higher risk $(26 \%)$ of being readmitted to hospital than with other diagnoses. ${ }^{25}$ Other frequent medical conditions for the elderly are stroke, diabetes, malnutrition and infections often occurring in addition to other chronic diseases, resulting in a compound treatment and care picture requiring integrated care.

Dementia is another common condition among the elderly. In Norway, 70000 elderly individuals were diagnosed with dementia in 2009. The incidence is close to $20 \%$ in the 80-84-year-old age group, increasing to approximately $40 \%$ for the $90+$ age group. ${ }^{22}$ A countrywide supervision (Norwegian Board of Health Supervision) of municipal health and social services for the elderly concluded that a lack of continuity exists in the care of the frail elderly with dementia. ${ }^{22}$

Based on the discussion thus far, the patient and next-of-kin inclusion criteria for the study are as follows:

- Elderly patients (>75 years old) receiving healthcare in the municipality (nursing home or home-based care services) with

- Hip fracture (upper femur) or

- COPD-related problems (eg, pneumonia, respiratory disorder)

- Patients with dementia admitted or discharged with one of the two diagnoses (upper femur hip fracture or COPD-related problems)
- Poly-pharmacy ( $>5$ medications)

- Next of kin for the patients meeting the above inclusion criteria.

Inclusion criteria might be adjusted if patient volume is limited. If that is the case, patients older than 70 years with other medical conditions (eg, stroke, diabetes, malnutrition, infections) than upper femur fracture and COPD-related problems will also be included. Poly-pharmacy and variation across orthopaedic and medical patients will be sought.

The main criterion for inclusion of healthcare personnel in the study has been their relation to the transitional care of the included patient group, as previously described. Healthcare personnel involved in admission or discharge, in community-based care (nursing homes or home-based services) or hospital services to be included in the study sample, include the following professional groups in case A and case B: (1) paramedics, (2) doctors at hospital, (3) doctors employed at nursing homes, (4) general practitioners, (5) nurses at hospital, (6) nurses in municipalities (nursing homes, homebased services), (7) physiotherapists at hospital, (8) physiotherapists in municipalities and (9) community-based patient coordinators. Variation in gender and experience among healthcare personnel will be sought.

\section{Data collection}

The study employs a triangulation of qualitative methods; ${ }^{26}$ participant observation constitutes the main part, supported by document analysis and followed by structured interviews. Table 1 displays the different qualitative methods employed in the study together with expected data materials in case $\mathrm{A}$ and case $\mathrm{B}$.

Data collection will be standardised across the two case study sites using an agreed observation guide and

Table 1 Data collection methods and material

\begin{tabular}{|c|c|c|}
\hline Methods & Case A (rural) & Case B (city) \\
\hline $\begin{array}{l}\text { Participant observation (including } \\
\text { open-ended, interactive } \\
\text { conversations with patients, next of }\end{array}$ & $\begin{array}{l}\text { Admission: 8-10 patient cases (mix of } \\
\text { orthopaedic and medical, } 2-5 \mathrm{~h} \text { of } \\
\text { observation per case) }\end{array}$ & $\begin{array}{l}\text { Admission: } 12-15 \text { patient cases (mix of } \\
\text { orthopaedic and medical, } 2-5 \mathrm{~h} \text { of } \\
\text { observation per case) }\end{array}$ \\
\hline kin, staff) & $\begin{array}{l}\text { Discharge: 8-10 patient cases (mix of } \\
\text { orthopaedic and medical, } 5-10 \mathrm{~h}^{\star} \text { of } \\
\text { observation per case) }\end{array}$ & $\begin{array}{l}\text { Discharge: } 12-15 \text { patient cases (mix of } \\
\text { orthopaedic and medical, } 5-10 \mathrm{~h}^{\star} \text { of } \\
\text { observation per case) }\end{array}$ \\
\hline \multirow[t]{2}{*}{ Individual interviews with staff } & $\begin{array}{l}\text { Admission: } 12-15 \text { (ambulance workers, } \\
\text { ER nurses, ER doctors) }\end{array}$ & $\begin{array}{l}\text { Admission: } 12-15 \text { (ambulance workers, } \\
\text { ER nurses, ER doctors) }\end{array}$ \\
\hline & $\begin{array}{l}\text { Discharge: } 20-30 \text { (hospital doctors, } \\
\text { general practitioners, nursing home } \\
\text { doctors, hospital nurses, nursing home } \\
\text { nurses, home-based care nurses, hospital } \\
\text { physicians, community-based physicians, } \\
\text { community-based patient coordinators) }\end{array}$ & $\begin{array}{l}\text { Discharge: } 20-30 \text { (hospital doctors, } \\
\text { general practitioners, nursing home } \\
\text { doctors, hospital nurses, nursing home } \\
\text { nurses, home-based care nurses, hospital } \\
\text { physicians, community-based physicians, } \\
\text { community-based patient coordinators) }\end{array}$ \\
\hline \multirow[t]{2}{*}{ Document analysis } & $\begin{array}{l}\text { Admission: admission summaries, } \\
\text { medication lists }\end{array}$ & $\begin{array}{l}\text { Admission: admission summaries, } \\
\text { medication lists }\end{array}$ \\
\hline & $\begin{array}{l}\text { Discharge: discharge summaries, } \\
\text { medication lists, follow-up care notes }\end{array}$ & $\begin{array}{l}\text { Discharge: discharge summaries, } \\
\text { medication lists, follow-up care notes }\end{array}$ \\
\hline
\end{tabular}


interview guide. One researcher (DND) will conduct observations and interviews related to admission in case $\mathrm{A}$ and case B while another researcher (KAL) will conduct observations and interviews related to discharge in case A and case B. The two researchers will conduct data collection simultaneously at the two case study sites and will meet regularly to discuss first impressions, review data collection tools and methods, and conduct possible follow-ups of data. The three data collection methods are described in more detail in the following sections.

\section{Participant observation}

Participant observation ${ }^{27}$ will be carried out related to admission and discharge transitions according to an agreed-upon observation guide based on several literature reviews. $^{7} 2829$ Themes in the observation guide include: (1) structures/plans, (2) coordination with other care providers, (3) conversation/coordination with patient and next of kin, (4) interdisciplinary collaboration, (5) documentation/information, (6) time factors, stress, other elements and (7) results. In addition, demographic data related to patients (age/gender, diagnosis, medications) and personnel (age/gender, position, work experience) will be noted. No tape-recording will be used during the observations due to the complexity of personnel, patient and next of kin involved. Observation summaries will be written consecutively. Observations include short conversations with all personnel involved in the transition and with the patients and next of kin.

Observation of admissions will start with the handover from the ambulance personnel to the nurses in the emergency room and end with a structured conversation with the patient at the ward 1 or 2 days after hospital admittance. The researcher will observe the interaction, coordination and dialogue (written in the forms of information and documentation developed and transferred and oral in the forms of communication) among the healthcare personnel (eg, paramedics, nurses, doctors), patient and next of kin. Copies of admission summaries and medication lists will be made during the participant observations. The observations on the day of admittance will conclude with short conversations with healthcare personnel asking them to clarify aspects of the current admission and evaluate the quality of the admission process.

Observations of discharges will start on the morning of the day of discharge (with the doctor's round) and end with structured conversations with the patient and involved personnel at the nursing home or in the homebased care services from 1 or 2 days after discharge and up to 30 days after discharge. The researcher will observe the interaction, coordination and dialogue (written and oral) among the healthcare personnel (eg, nurses, doctors, physiotherapists), patient and next of kin on the day of discharge. Copies of discharge summaries and medication lists will be made during the participant observation. The researcher will, if possible, observe the patient on arrival at the nursing home or home with home-based care services and conduct a follow-up observation 1 or 2 days after discharge. During the follow-up observation, copies of written documentation related to follow-up care (care plans, medical cards, doctors' and nurses' notes) will be made, if available. During discharge observations, short conversations with nurses and physicians at hospital and in primary care will be conducted to clarify aspects of the current discharge and evaluate the quality of the discharge process. If possible, some of the patients will be observed up to 30 days after discharge to map follow-up care, readmissions and transitions between short-term and long-term rehabilitation institutions. The follow-up observations will include mapping of where the patients stay during the 30-day period, the care providers involved and short conversations with the patients, next of kin and personnel (face to face or via the phone).

\section{Individual interviews}

As part of the participant observations, short-ended and open-ended individual interviews will be conducted with the observed patients and next of kin 1 or 2 days after admission to and discharge from hospital. The interviews will be in the form of unstructured and interactive conversations with the aim of capturing interviewees' experiences of transitional care, as well as perspectives and stories related to the observed admission or discharge process. Themes to be covered in the patient and next-of-kin conversations include (1) transition process, (2) preparation/preparedness, (3) involvement/participation, (4) information, (5) interdisciplinary collaboration, (6) satisfaction, (7) incidents and (8) improvements. During observations of discharge transitions, several conversations might take place during the period of up to 30 days of follow-up care.

After finishing the participant observation data collection, structured interviews will be conducted with personnel in primary and secondary healthcare services involved in admissions and discharge processes following an agreed-upon interview guide. Themes to be covered in the personnel interviews include (1) coordination/ interaction among care providers (experiences, success, insufficiency, improvements), (2) multidisciplinary collaboration, (3) information exchange, (4) knowledge sharing, (5) quality and safety, (6) patient and family involvement/education, (7) structure/planning and (8) challenges/barriers. The structured interviews will build on the participant observations as the same researcher will conduct the observations and interviews. Although no detailed analysis of observational data will exist at the time of the structured interviews, the researcher will have conducted a rough 'first impression' analysis based on observational notes and summaries, giving her the possibility to pick up on important issues after being in the field of transitional care. This approach will provide her with an important contextual 
understanding that will enable her to give the structured interviews more depth and examples on which to build the conversation. The structured interviews of personnel in case A and case B will build on a saturation principle, ${ }^{27}$ meaning that the number of interviews will be adjusted according to the amount of accumulative information they bring.

\section{Document analysis}

Admission and discharge summaries, medication lists and written documentation related to admission and follow-up care will be copied during the participant observations at hospitals, nursing homes and homebased nursing care settings. The documentation will act as important data material to be used in follow-up conversations and interviews related to each of the observed patients and in general to evaluate the quality of written documentation.

Registered adverse events related to coordination issues (mandatory field in the registration system) will be analysed for the 2008-2012 period according to frequency. A selection of events will be analysed in detail according to types, causes and topics. The documented events will be used to inform research question $\mathrm{C}$ and a detailed analysis of the risks involved in transitional care in case A and case B.

\section{Data analysis}

To ensure trustworthiness in the analysis, we will apply analyst triangulation and member checks. ${ }^{30} 31$ Our research team will discuss and refine the analysis according to our research questions and themes emerging in the data. All transcribed observations and interviews will be uploaded and systematised using Nvivo.

A 'big picture' or total impression of the data material will first be created using Malterud's step 1 in a systematic text condensation approach. ${ }^{32} 33$ This will involve the entire research team and an external researcher not involved in the design of the study. Researchers in the research team will individually read the data material and discuss the overall emerging themes. After agreeing on a set of themes, the research team will meet with the external researcher who has individually created his/her overall themes to discuss and agree on a common set of themes to be included in the 'big picture'. It is this total impression that will form the basis for the development of phase 2 of the 'Quality and Safety in Transitional Care of the Elderly', the evidence-based intervention programme, and that will inform research questions A, $\mathrm{B}$ and $\mathrm{E}$.

Step 2 of the qualitative data analysis will involve in-depth analyses of two specific themes. This will involve the risk perspective in transitional care (research question $\mathrm{C}$ ), carried out by KAL as the principal analyst, and the patient perspective in transitional care (research question $\mathrm{D}$ ), carried out by DND as the principal analyst. These analyses will involve creating subthemes, categories and meaning units $^{32}$ as well as applying different theoretical perspectives (patient participation, risk/resilience) to the data material and including researcher triangulation with the rest of the research team.

After analysing the data from observations, interviews and documents, member checks ${ }^{30}{ }^{31}$ will be conducted in two focus groups (one hospital based and one community based) and in one workshop (common for hospital-based and community-based services) to validate the research findings and involve the participants in possible intervention measures (phase 2) in each of the two cases (a total of four focus groups and two workshops). All participants in phase 1 of the 'Quality and Safety in Transitional Care of the Elderly' study will be invited to attend the workshops. The focus groups will consist of five to seven participants included in the study covering admission and discharge at the hospital and nursing homes and home-based care services in the municipalities.

\section{Ethical concerns and dissemination}

The study has been approved by the Norwegian Regional Committees for Medical and Health Research Ethics (REC, no. 2011/1978).

The study is based on informed written consent, and informants can withdraw from the study at any point in time. Interview and observation data material will be managed confidentially (indirect person identifiable). Tape recordings will be deleted immediately after transcription. Each transcribed interview and observation will be marked with a code, and the list matching the person identification and code will be securely stored (locked cabinet or password-protected PC at the university) by the research group (principal researcher). Transcribed data material will be stored at the research institution for 3 years after the project ends. Paper copies of admission and discharge summaries, medication lists and written documentation related to follow-up care (if available) will be made, deleting direct person-identifiable information and any information not relevant for this study. Thus, superfluous information (eg, previous medical conditions) will not be included. Copies will be made and signed by the responsible nurse, checking that all direct person-identifiable information and access information are deleted. The copies of patient-related summaries will be stored in a locked cabinet at the research institution.

Results are to be disseminated at several congresses and research conferences and in articles published in peer-reviewed journals. In addition, we are going to present study results to people outside the academic community through public presentations.

\section{DISCUSSION}

Real-time observation of transitions involving patients crossing care provider and care-level boundaries is a complex endeavour. It involves data collection in 
multiple locations, with multiple professions, patients and next of kin. Transitional care is furthermore a complex phenomenon involving a substantial set of dimensions or factors (eg, patient involvement, coordination, multiprofessional collaboration, documentation, information or communication) to be explored and mapped. As a whole, this complexity forces fierce prioritisations on the design of a study of transitional care of the elderly. In this study, prioritisations have been taken along the axes of the patient groups included (hip fracture and COPD, with or without dementia), the cases included (one rural and one city based) and the researchers included (single researchers on admission and discharge, respectively, but with analyst triangulation).

The main contribution of the study will be contextual knowledge created by real-time observations of transitional care practices of the elderly crossing care provider boundaries. This adds to the existing literature primarily concerned with individual and focus group interviews with professionals and/or patients.

Undoubtedly, several limitations exist in terms of the design of the 'Quality and safety in Transitional Care of the Elderly' project due to the complexity of the scope and aims of the study. Most of the limitations are caused by practical and resource-based constraints and obstacles. One of the limitations concerns data collection at admission, where observation start is set in the emergency room at the handover from the ambulance personnel to the ER nurse. Ideally, data collection on admission transitions from primary care to secondary care would benefit from starting at the nursing home or home with home-based care services and following the patient on the day of admission. This has not been possible to incorporate into the current project due to the size of the study and practical problems with recruiting these patients and their care providers.

In addition, it would have been valuable for the study to acquire data on the entire elderly patient pathway, from the day of admission through hospital stay (with belonging transitions) through discharge and 30-day follow-up care. As the 'Quality and Safety in Transitional Care of the Elderly' study is designed, data from hospital stay are restricted to the number of days hospitalised. Another limitation relates to the challenges of data collection in case A (rural). Owing to its rural location, practical and resource-based issues (eg, travel costs for researchers) will create obstacles for the depth and length of data collection activities. In particular, this will influence the possibility to do follow-up observations up to 30 days after discharge.

Finally, a note on the possible observer bias is worth mentioning as we have chosen to conduct the observations with single researchers (both having a nursing background), doing the observations and interviews in admission and discharge transitions. Ideally, an observation team with a minimum of two researchers with different backgrounds could better cover the complexity of the observation setting involving the professional as well as the patient perspectives. We tried to control the observer bias by setting up the observations of admission and discharge at the same point in time so that the regular exchange of fieldwork impressions between the two researchers could take place. In one or two observations, we will include an additional researcher from the research team to validate the observation summaries. In addition, we have set up weekly meetings or updates in the observational periods with the entire research team (backgrounds within nursing, management, and quality and safety) to debrief and discuss preliminary impressions. Regular discussions among the research group and a wider international advisory board will also provide opportunities for reflexivity and the development of insights into fieldwork and data analysis.

Despite the limitations of the study design, we argue that the uniqueness of our study design applied to explore different aspects of transitional care is considerable. Thus far, a limited number of studies have applied participant observation in order to grasp the context and complexity surrounding transitional care. The use of the triangulation of methods to increase the credibility of the research findings ${ }^{26}$ and the focus on the frail elderly identified as a particularly vulnerable group in transitional care ${ }^{34}$ represent other novel aspects of this study. The application of professional-centred and patient-centred perspectives will generate new and increased understanding within the field of transitional care of the elderly. The study results will furthermore be used to guide the design of an intervention programme with specific measures to be implemented to ensure quality and safety in the transitional care of the elderly across primary and secondary care (phase 2 ).

Acknowledgements We would like to thank the two funding organisations for the possibility they have given us for carrying out research on what we value as an important topic. We also want to express our gratitude to Berit Tjørhom for her contribution in the grant application phase and to Oliver Groene for his comments on an earlier draft of this study protocol manuscript.

Contributors KA developed the original proposal for grant application, planned the study design and the study protocol, contributed to the development of data collection tools and drafted and revised this manuscript. KAL contributed to the study design, was responsible for the development of data collection tools and ethical approval, and contributed to the drafting and revision of the manuscript. DND contributed to the development of data collection tools and commented on the study protocol and manuscript draft and revision. MS commented on the study protocol and data collection tools, and contributed to the manuscript draft and revision. All authors read and approved the final manuscript.

Funding The study 'Quality and Safety in Transitional Care of the Elderly' has received funding from the Norwegian Research Council (under the title 'Quality and Safety in Elderly Health and Care Services-the role of interactions and transitions') under grant agreement no. 204637 and from the Western Norway Regional Health Authority under project no. 911642.

Competing interests None.

Patient consent Obtained.

Ethics approval The Norwegian Regional Committees for Medical and Health Research Ethics.

Provenance and peer review Not commissioned; internally peer reviewed. 
Open Access This is an Open Access article distributed in accordance with the Creative Commons Attribution Non Commercial (CC BY-NC 3.0) license, which permits others to distribute, remix, adapt, build upon this work noncommercially, and license their derivative works on different terms, provided the original work is properly cited and the use is non-commercial. See: http:// creativecommons.org/licenses/by-nc/3.0/

\section{REFERENCES}

1. Coleman EA, Boult C. Improving the quality of transitional care for persons with complex care needs. J Am Geriatr Soc 2003;51:556-7.

2. Coleman EA. Falling through the cracks: challenges and opportunities for improving transitional care for persons with complex care needs. J Am Geriatr Soc 2003;51:549-55.

3. Tsilimingras $D$, Rosen AK, Berlowitz DR. Patient safety in geriatrics: a call for action. J Gerontol 2003;58:813-19.

4. Naylor M, Keating SA. Transitional care. Am J Nurs 2008;108:58-63.

5. Garaasen $\mathrm{H}$, Johnsen $\mathrm{R}$. The quality of communication about older patients between hospital physicians and general practitioners: a panel study assessment. BMC Health Serv Res 2007;7:133.

6. LaMantia MA, Scheunemann LP, Viera A, et al. Interventions to improve transitional care between nursing homes and hospitals: a systematic review. JAGS 2010;58:777-82.

7. Laugaland KA, Aase K, Barach $\mathrm{P}$. Addressing risk factors for transitional care of the elderly-literature review. In: Albolino, Bagnara, Bellandi, Llaneza, Rosal, Tartaglia, eds. Healthcare systems ergonomics and patient safety. London: Taylor \& Francis Group, 2011:161-2.

8. Tsilimingras D, Bates DW. Addressing post-discharge adverse events: a neglected area. Jt Comm J Qual Patient Saf 2008;34:85-97.

9. Johnson J, Barach P, Vernooij-Dassen M. Conducting a mulitcentre and multinational qualitative study on patient transitions. BMJ Qual Saf 2012;21(Suppl 1):i22-8.

10. Hesselink G, Flink M, Olsson M, et al. Are patients discharged with care? A qualitative study of perceptions and experiences of patients, family members and care providers. BMJ Qual Saf 2012;21(Suppl 1): i39-49.

11. Flink $\mathrm{M}$, Ohlen $\mathrm{G}$, Hansagi $\mathrm{H}$, et al. Beliefs and experiences can influence patient participation in handover between primary and secondary care-a qualitative study of patient perspectives. BMJ Qual Saf 2012;21(Suppl 1):i76-83.

12. Flink M, Hesselink G, Pijnenborg L, et al. The key actor: a qualitative study of patient participation in the handover process in Europe. BMJ Qual Saf 2012;21(Suppl 1):i89-96.

13. Groene RO, Orrego C, Sunol R, et al. "It's like two worlds apart": an analysis of vulnerable patient handover practices at discharge from hospital. BMJ Qual Saf 2012;21(Suppl 1):i67-75.

14. Siemsen IMD, Madsen MD, Pedersen LF, et al. Factors that impact on the safety of patient handovers: an interview study. Scand J Public Health 2012;40:439-48.

15. Flin R, Mitchell L,. eds. Safer surgery. Analysing behaviour in the operating theatre. Ashgate: Surrey, 2009.
16. Abraham J, Kannampallil TG, Patle VL. Bridging gaps in handoffs: a continuity of care based approach. J Biomed Informatics 2012;45:240-54.

17. Lyhne S, Georiou A, Marks A, et al. Towards an understanding of the information dunamics of the handover process in aged in care settings - a prerequisite for the safe and effective use of ICT. Int $J$ Med Informatics 2012;8:452-60.

18. DiGioia A, Greenhouse KP. Patient and family shadowing. JONA 2011;41:23-8.

19. Yin RK. Case study research: design and methods. 3rd edn. Applied social research methods series. Thousand Oaks: SAGE Publication, 2003.

20. Gurner U, Thorslund M. Holistic perspective is needed in care of multiimpaired elderly. A proposal for structural change of the organization of care in the county of Stockholm. Läkartidningen 2001;98:2596-602.

21. The Norwegian Prescription Database 2004-2008 Medication Statistics 2009:2. Oslo: Norwegian Institute of Public Health, 2009. http://www.fhi.no/dokumenter/197a20d501.pdf (accessed 22 Dec 2012).

22. Norwegian Board of health Supervision. Complex tasks and services require stronger governance. Summary report of countrywide supervision in 2010 of municipal health and social services for elderly people. Report no. 5/2011.

23. Nickelsen T. Venter for lenge på operasjon: Eldre med hoftebrudd får delirium. Norway: Research Magazine Apollon, University of Oslo, 2010. http://www.apollon.uio.no/artikler/2010/delirium.html (accessed 22 Dec 2012).

24. World Health Statistics (2008).

25. Lindman AS, Damgaard K, Tjomsland O, et al. Re-admissions of elderly in Norway. Oslo: The Norwegian Knowledge Centre for the Health Services (NOKC), 2012

26. Lincoln YS, Guba EG. Naturalistic inquiry. London: Sage Publication, 1985

27. Polit DF, Beck CT. Nursing research: generating and assessing evidence for nursing practice. 8th edn. Philadelphia, PA: Wolters Kluwer/Lippincott/Williams \& Wilkins, 2008.

28. Laugaland KA, Aase $\mathrm{K}$, Barach $\mathrm{P}$. Interventions to improve patient safety in transitional care-a review of the evidence. Work 2012;41:2915-24

29. Dyrstad DN, Testad I, Aase K, et al. A systematic review of the literature on patient participation in transitions of the elderly. Cognition: Technology \& Work, 2013, Special Issue. In press.

30. Lincoln YS, Guba EG. Naturalistic inquiry. Beverly Hills, CA: Sage, 1985

31. Seale C. The quality of qualitative research. London: Sage, 1999

32. Malterud K. Kvalitative metoder i medisinsk forskning. Universitetsforlaget, 2003.

33. Malterud K. Systematic text condensation: a strategy for qualitative analysis. Scand J Public Health 2012;40:795-805.

34. Coleman EA, Min SJ, Chomiak A, et al. Posthospital transitions: patterns, complications, and risk identification. Health Serv Res 2004;39:1449-65. 